\title{
Virtudes y fortalezas del carácter en población adulta de Buenos Aires. Un estudio con un enfoque mixto émico/ético
}

\author{
Virtues and character strengths in Buenos Aires adult population. \\ A study with a mixed emic/etic approach
}

\author{
Alejandro Castro Solano* \\ Alejandro C. Cosentino \\ Universidad de Palermo, Argentina \\ (Rec.: mayo de 2016 - Acept.: marzo de 2017)
}

\section{Resumen}

El presente estudio tiene como objetivo establecer la validez cultural de la clasificación VIA (24 fortalezas en 6 virtudes) mediante la utilización de un enfoque mixto (ético/émico). Se intentó determinar si la clasificación VIA resultaba relevante culturalmente para poder capturar las respuestas de personas legas en ocasión de describir las características positivas de personajes protípicos admirados en un formato de respuesta libre (free listing). Participaron del estudio una muestra de 378 personas adultas de población general no consultante argentina, varones y mujeres. Los resultados señalan que las fortalezas más frecuentes fueron la integridad, la persistencia, la bondad y el amor, lo que da lugar a dos perfiles diferenciados de personajes prototípicos de características positivas. No se encontraron diferencias según sexo y edad. Los resultados sugieren que la clasificación VIA debe ser ajustada en función de las variables culturales.

Palabras clave: psicología positiva, fortalezas del carácter, rasgos positivos.

\begin{abstract}
The purpose of the present study is to establish the cultural validity of the Values in Action (VIA) classification through the use of 24 character strengths contained in 6 virtues, by implementing a combined emic-etic approach. A group of 378 male and female Argentinian adults participated from this research. The aim was to determine if the VIA classification was culturally relevant to portray the answers of the participants involved. Results indicate that the most frequent strengths listed by the participants were integrity, persistency, kindness and love. No differences regarding age and gender were found. The results also suggest that the VIA classification needs to be adjusted according to cultural variables.
\end{abstract}

Keywords: positive psychology, character strengths, positive traits, virtue, ethnic values.

\footnotetext{
Correspondencia a: Alejandro Castro Solano, Ph.D., Investigador independiente del Consejo Nacional de Investigaciones Científicas y Técnicas (CONICET). Dirección: Paraguay 5337, 50 "A", Buenos Aires, Argentina, C1425BTI. Teléfono: (11) 4771 6029. E-mail: alejandro.castrosolano@gmail.com.
} 


\section{Introducción}

Hacia finales de la década de los noventa, Martin Seligman proponía que la psicología no solo era el estudio de la enfermedad, la debilidad y el daño, sino también el estudio de las fortalezas de carácter y de las virtudes, las cuales pueden ser cultivadas para mejorar la calidad de vida de las personas (Seligman \& Csikszentmihalyi, 2000). Seligman propuso un nuevo campo de trabajo que denominó Psicología Positiva (PP en adelante), cuyo objetivo fue reorientar la ciencia psicológica y la práctica clínica con el objetivo de identificar los aspectos que contribuyeran a la salud psicológica y que permitieran desarrollar los rasgos positivos de los jóvenes. Los autores de este movimiento propusieron utilizar rigurosas técnicas de la investigación científicas para poder sentar las bases de este modelo (Fowler, Seligman \& Koocher, 1999).

La PP considera tres pilares principales: el subjetivo, el individual y el institucional. El pilar subjetivo explora las experiencias subjetivas que son evaluadas positivamente, tales como las emociones positivas, la satisfacción con la vida y el bienestar psicológico. El área individual comprende los rasgos individuales positivos, las virtudes y fortalezas de carácter. El pilar institucional estudia los grupos y organizaciones con un fuerte énfasis en los aspectos positivos de los individuos que conforman esos grupos. En particular, el estudio del segundo pilar de PP - fortalezas del carácter y virtudes - es importante para promover el bienestar de las personas (Park, Peterson \& Seligman, 2004) y tiene un papel central en este enfoque (Park \& Peterson, 2009), ya que dichos rasgos positivos son el pivote entre el bienestar y las instituciones positivas.

Peterson y Seligman (2004) propusieron el estudio de las fortalezas del carácter utilizando una metodología científica. Así, argumentaron que la clasificación de las fortalezas de carácter era un paso importante y necesario hacia el estudio científico de excelencia moral. Estos investigadores desarrollaron una clasificación de las virtudes que se constituiría en la base para el estudio empírico de este constructo y para el desarrollo de las intervenciones psicológicas. Dahlsgaard, Peterson y Seligman (2005) propusieron que 6 virtudes aparecían implícita o explícitamente mencionadas en algunos textos tradicionales filosóficos y religiosos de oriente (p. ej., hinduismo) y occidente (p. ej., filosofía ateniense). Luego de análisis y debates académicos, los autores propusieron un listado de 24 fortalezas del carácter que se correspondían con esas virtudes. Esta clasificación de 6 virtudes y 24 fortalezas ha tenido un uso extendido dentro de los autores de la PP (Ver la Tabla 1).

Tabla 1. Clasificación "Values in Action" de 24 Fortalezas en 6 Virtudes de Peterson y Seligman (2004)

\begin{tabular}{|c|c|c|}
\hline & Fortaleza & Definición resumida \\
\hline \multirow[t]{3}{*}{ Justicia } & Imparcialidad & Hacer juicios sociales equitativos \\
\hline & Liderazgo & Guiar al grupo en armonía hacia el éxito \\
\hline & Ciudadanía & Comprometerse con el grupo social \\
\hline \multirow[t]{3}{*}{ Humanidad } & Bondad & Ayudar a todos, sin fines utilitarios \\
\hline & Amor & Buscar estar cerca de los afectos de uno \\
\hline & Inteligencia Social & Saber lo que desean y buscan los demás \\
\hline \multirow[t]{5}{*}{ Sabiduría y conocimiento } & Perspectiva & Juicio elevado y profundo sobre la vida \\
\hline & Creatividad & Tener ideas originales y útiles \\
\hline & Apertura Mental & Buscar visiones alternativas \\
\hline & Amor por el saber & Buscar más y mejores conocimientos \\
\hline & Curiosidad & Deseo vivo de experimentar y conocer \\
\hline \multirow[t]{5}{*}{ Trascendencia } & Espiritualidad & La vida tiene un sentido más allá de uno \\
\hline & Gratitud & Sentir y expresar agradecimiento \\
\hline & Esperanza & Estar convencido de que todo saldrá bien \\
\hline & Humor & Visión alegre y serena de la vida \\
\hline & Apreciación & Emocionarse con lo excelso \\
\hline
\end{tabular}




\begin{tabular}{lll}
\hline Templanza & Clemencia & Tornarse benévolo hacia el trasgresor \\
& Autorregulación & Gobernar las respuestas a los estímulos \\
& Prudencia & Tomar las decisiones cuidadosamente \\
& Humildad & Dejar que los propios logros hablen por sí \\
\hline Coraje & Valentía & Hacer lo correcto, corriendo riesgos \\
& Integridad & Practicar lo que se predica \\
& Persistencia & Concluir las tareas, pese a los obstáculos \\
& Vitalidad & Sentirse vivo y efectivo
\end{tabular}

Nota. Apreciación = Apreciación de la belleza y de la excelencia. Ciudadanía = Ciudadanía o trabajo en equipo, Clemencia $=$ Clemencia y misericordia. Humildad $=$ Humildad/Modestia.

Desde esta perspectiva se intentó a largo de la última década diseñar y validar una clasificación de las virtudes y fortalezas humanas (Values in Action [VIA]), que fuese el "reverso" del Manual Diagnóstico y Estadístico de los Trastornos Mentales y que opere como un verdadero "Manual de las sanidades". Peterson y Seligman (2004) han afirmado que la clasificación VIA se desarrolló con la esperanza de impulsar y generar un lenguaje común para los estudios científicos en PP.

Los estudios empíricos inspirados en esta clasificación mostraron que las fortalezas del carácter estaban asociadas con diferentes variables de interés, como sexo y edad (Linley, Joseph, Harrington \& Wood, 2006), genética (Steger, Hicks, Kashdan, Krueger \& Bouchard, 2007), grupos sociales (Cosentino \& Castro Solano, 2012), satisfacción con la vida (Park, Peterson \& Seligman, 2004), personalidad (Macdonald, Bore \& Munro, 2008), rendimiento académico en estudiantes universitarios (Lounsbury, Fisher, Levy \& Welsh, 2009), rendimiento académico y militar en estudiantes militares (Cosentino \& Castro Solano, 2012), recuperación de enfermedades (Peterson, Park \& Seligman, 2006) y crecimiento postraumático (Peterson, Park, Pole, D’Andrea \& Seligman, 2008), entre otros.

Sin embargo, a pesar de que Peterson y Seligman (2004) utilizaron la cuestión cultural como criterio base del desarrollo de la clasificación VIA, como se hace evidente por el estudio sobre textos de diferentes tradiciones culturales para hacer de basamento de su clasificación, ninguna de las 24 fortalezas y ninguna de las seis virtudes refiere centralmente a cuestiones culturales (Cosentino \& Castro Solano, 2014; Dahlsgaard et al., 2005). Tampoco han proliferado estudios empíricos que, utilizando la clasificación VIA, hubiesen analizado la relación entre los rasgos del carácter y aspectos psicológicos culturales.

Por otra parte, Peterson (2006) afirmaba que esta clasificación resultaba tentativa y que se encontraba sujeta a los cambios consecuentes de la profundización en el estudio científico de la excelencia moral, siendo esperable que la misma sea revisada e incluso modificada. En este sentido, podrían aparecer nuevas fortalezas, combinarse o desaparecer algunas de las definidas. Asimismo, podrían existir fortalezas del carácter que solo son consideradas como tales en unas culturas, pero no en otras (Lambert, Pasha-Zaidi, Passmore, \& AlKaram, 2015; Peterson \& Park, 2009; Snyder, Lopez, \& Pedrotti, 2011). De la misma forma, podían existir fortalezas características de una población que no hayan sido incluidas en la clasificación original VIA comentada (Peterson, 2006).

La equivalencia cultural de los constructos positivos ha sido una preocupación desde los inicios del movimiento (López et al., 2002). El debate acerca de las virtudes y fortalezas humanas toma en cuenta dos aspectos contrarios. Por un lado, se encuentra la línea que sostiene que las fortalezas son universales, que la clasificación diseñada por Peterson y Seligman es aplicable a la mayoría de las culturas y que por lo tanto sería cultural free. La línea contraria sostiene que, si bien algunas de las fortalezas pueden estar presentes en varias culturas distintas, los investigadores deben estar alerta ante posibles sesgos etnocéntricos al manejarse la clasificación solo desde la perspectiva éticoimpuesta (Christopher \& Hickinbottom, 2008; Snyder et al., 2011).

Aunque se han realizado varios estudios con poblaciones de diferentes orígenes culturales con el fin de explorar la variación cultural de los constructos de la PP, la perspectiva más utilizada para el estudio de las fortalezas ha sido la ético-impuesta (Berry, 1969; Triandis, Malpass \& Davidson, 1973). Este enfoque supone un sistema descriptivo que sea igualmente válido para todas las culturas estudiadas y que permita la representación de similitudes y diferencias entre ellas (Helfrich, 1999). Por lo tanto, los estándares para hacer la comparación deben ser equivalentes en todas las culturas (en nuestro caso la clasificación de fortalezas). Este enfoque incurre en el supuesto de utilizar instrumentos émicos como si fueran éticos (válidos universalmente). De manera contraria, el enfoque émico considera los fenómenos psicológicos desde la perspectiva de las personas y evalúa el comportamiento desde una cultura en particular, utilizando los conceptos más adecuados 
para esa cultura específica (Davidson, Jaccard, Triandis, Morales \& Díaz-Guerrero, 1976).

En consecuencia, los riesgos de utilizar un enfoque ético son el ignorar conceptos e ideas propios de esa cultura. Por el contrario, utilizar solamente la perspectiva émica podría obstaculizar la investigación y la comparación transcultural llevando la psicología a una variante nativa o indigenista (Berry, 1989; Berry, Poortinga, Segall \& Dasen, 2006; Kagitcibasi \& Berry, 1989). Ambos enfoques, en sentido estricto, resultan problemáticos. Los autores culturales han propuesto soluciones al dilema émico/ético. Una solución es tomar un enfoque ético impuesto (top-down) que fue el comentado recientemente y el que emplean la mayoría de los psicólogos positivos en el estudio de las fortalezas humanas. El riesgo de este enfoque es que las investigaciones propongan "falsos universales". Una perspectiva intermedia (éticoderivada) es la adoptada en este trabajo (Berry et al., 2006; Sinha, 1997). Se comienzan estudiando instrumentos y constructos éticos, en primer lugar, y, en segundo, lugar se verifica cuáles elementos son adecuados y cuáles no a la cultura específica que se estudia. Esta aproximación permite verificar qué aspectos de los constructos psicológicos son realmente universales y cuáles son específicos o propios de cada cultural en particular.

Cabe destacar el escaso desarrollo, en los países hispanoparlantes, de los constructos más modernos sobre los que la PP hace foco: las fortalezas, las emociones positivas, las organizaciones positivas, etc. que resultan ser temas de escaso interés y están subrepresentados en las producciones científicas de los psicólogos latinos (Castro Solano, 2014). En estos estudios, en términos generales, se descuida la perspectiva cultural-regional, dando por sentada la supuesta universalidad de los constructos psicológicos. Se extraen conclusiones acerca de poblaciones muy específicas (e.g, estudiantes universitarios, poblaciones anglosajonas, de raza blanca, de nivel socioeconómico medio, etc.) y se extrapolan estos resultados a contextos culturales más amplios, sin un estudio adecuado de las variaciones locales de los constructos estudiados. La suposición de la universalidad, partiendo de una base de datos limitada, constituye no solo un problema teórico, sino también empírico, ya que se establecen estrategias de intervención en los ámbitos de aplicación tomando como referencia investigaciones con sesgos culturales evidentes (Casullo \& Fernández, 2006).

La adopción de esta estrategia top-down hace que se desvanezcan las variantes émicas locales, quedando estas subrepresentadas. Esto lleva a que los constructos psicológicos estudiados revistan características pseudoéticas o que en suma sean "falsamente universales". En los trabajos de PP revisados en la última década se destaca el poco interés que reviste el estudio de las variaciones locales de los constructos positivos diseñados generalmente en Estados Unidos y en Europa y la escasa importancia dada a los estudios transculturales o intraculturales, que se hallan en términos generales subrepresentados (Castro Solano, 2014). El presente estudio tuvo como objetivo establecer la validez cultural de la clasificación VIA mediante la utilización de un enfoque mixto (ético/émico). Se intentó determinar si la clasificación VIA resultaba relevante culturalmente para poder capturar las respuestas de personas legas con ocasión de describir las características positivas de personajes protípicos admirados en un formato de respuesta libre (free listing).

\section{Métodos}

\section{Participantes y Procedimiento}

Participaron de este estudio 378 adultos residentes en la Ciudad de Buenos Aires; 185 hombres (49\%) y 193 mujeres (51\%). La edad media fue de 41,4 años $(D T=13.1)$. La muestra estaba balanceada por sexo y edad. En cuanto a la educación, el $66 \%(n=251)$ de la muestra tenía estudios universitarios y el $31 \%$ $(n=116)$ había terminado la escuela secundaria. Se trataba de una muestra de población general altamente educada. En cuanto al estado civil, el 56\% $(n=212)$ estaba involucrado en una relación y el $28 \%(n=107)$ estaba soltero/a. La participación fue voluntaria y anónima y no se proporcionaron incentivos.

Dada la naturaleza cualitativa del estudio se solicitó a los participantes que llevaran a cabo dos tareas: (a) que indicaran el nombre de una persona admirada, excluyendo características físicas 0 económicas; asimismo, debían consignar qué tipo de personaje se trataba (p. ej. pariente, personaje histórico, de la televisión); (b) debían describir alguna situación imaginada o real en la que se pueda apreciar las cualidades personales de un personaje admirado, centrándose en las fortalezas personales de ese personaje.

\section{Análisis de Contenido}

Se llevó a cabo análisis de contenido con el fin de identificar (a) los tipos de personajes admirados, (b) las características personales de los personajes en cuestión (fortalezas); (c) la temática de la historia y (d) el tipo de vínculo o relación incluida en la historia.

\section{Personajes Admirados}

Las respuestas se agruparon en ocho categorías diferentes, tomando en cuenta los siguientes criterios: (a) los políticos / militares: los individuos reconocidos por sus posiciones o acciones políticas y militares (p. ej. Napoleón); esta categoría incluye a las personas que han sido servidores públicos durante algún período de su vida (p. ej., Barack Obama); (b) líderes espirituales o religiosas: se refiere a las personas con cargos eclesiásticos 0 reconocidas como representantes de las corrientes religiosas o espirituales (p. ej., el Papa); (c) población general: se incluyen a personas no conocidas o con algún vínculo con el participantes (p. ej., padre, jefe, amigo); (d) los líderes revolucionarios: los individuos principalmente reconocidos por su actividad revolucionaria, entendida como acciones contra el régimen establecido (p. ej., Martin Luther King); (e) los 
científicos: los sujetos reconocidos por sus trabajos científicos (p. ej., Albert Einstein); (f) artistas: músicos, pintores, actores, escritores y personajes asociados a las actividades culturales (p. ej., Salvador Dalí); (g) atletas: los individuos asociados con los deportes (p. ej., Roger Federer); (h) emprendedores: personas reconocidas por su desarrollo empresarial (p. ej., Steve Jobs).

\section{Características Personales de los Personajes Admirados (Fortalezas)}

Dos jueces expertos codificaron las palabras (características de los personajes admirados) tomando como base la clasificación VIA de 24 fortalezas. El primer paso fue determinar si cada una de las palabras podía ser representada por una fortaleza o era un sinónimo de ella. Por ejemplo, fueron codificadas como dentro de la fortaleza perseverancia, las palabras perseverancia, como así también constancia, tenacidad y empeño, ya que se consideraban todas palabras con significado equivalente entre sí. Si las palabras tenían una connotación negativa no fueron consideradas. Este procedimiento fue realizado de forma independiente por dos jueces psicólogos y revistió un alto grado de concordancia entre ambos juicios (Kappa > .70).

\section{Temas y Tipos de Relaciones en las Historias}

Un procedimiento similar se siguió para la codificación de las historias con el fin de identificar las fortalezas de la clasificación VIA que aparecían en los textos proporcionados por los participantes. Asimismo, se realizó un análisis de contenido sobre el tipo de vínculo indicado en la historia.

\section{Resultados}

Análisis de las Fortalezas en Prototipos Frecuencia de las Fortalezas en Prototipos

Como primer paso, se analizaron las palabras (las características admiradas positivas) utilizadas para describir los personajes prototípicos admirados. Se obtuvieron un total de 1134 características personales, de las cuales 147 respuestas (13\%) no se pudieron clasificar. Las palabras tales como fuerza, personalidad, directo, fuerte no pudieron ser clasificadas mediante la clasificación VIA de fortalezas. Respecto de la palabra inteligencia, ésta aparece 59 veces y tampoco resultó clasificable, dada la naturaleza genérica de la palabra. Por lo tanto, un total de 928 palabras $(81,8 \%)$ pudieron ser clasificadas correctamente utilizando la clasificación VIA de Peterson y Seligman. En cuanto a las historias, solo 19 no se pudieron clasificar, lo que constituye menos del $0.05 \%$ del total. Podemos decir que la clasificación VIA cubre prácticamente todos los atributos reconocidos tanto en personajes como en las historias narradas por los participantes.

En un segundo paso, se calcularon las frecuencias de ocurrencia de cada una de las fortalezas. Como cada participante podía caracterizar hasta con 3 palabras a cada uno de los personajes, se asumió una clasificación de respuesta dicotómica múltiple.
Las fortalezas más mencionadas (entre el 20\% y el $30 \%$ de menciones) fueron las relacionadas con la virtud del coraje (la integridad, la persistencia, la valentía), seguido de los rasgos asociados a la humanidad (la bondad y el amor). Por lo tanto, el perfil típico de las personas admiradas se puede caracterizar por la integridad, la persistencia en la consecución de sus objetivos, la valentía frente a la adversidad, la capacidad para ayudar a los demás y el amor por los seres queridos. Las fortalezas relacionadas con la espiritualidad, el trabajo en equipo, la inteligencia social, el perdón, la curiosidad y la apreciación de la belleza y de la excelencia no aparecieron en ninguno de los personajes $(<1 \%$ de menciones). Datos completos en Tabla 2.

\section{Agrupación de las Fortalezas en Prototipos Admirados}

Con el objetivo de identificar la agrupación de las fortalezas entre sí, se llevó a cabo un análisis de correspondencias múltiples. Este es un procedimiento alternativo al análisis factorial exploratorio para analizar variables discretas que permite el escalado de las variables categóricas y analizar las relaciones entre ellas. Se incluyeron en el análisis las fortalezas con una frecuencia de ocurrencia mayor al 5\%. Se obtuvieron dos dimensiones (ver Figura 1). La primera dimensión representó el $45 \%$ de la inercia total y permite apreciar un continuo entre las fortalezas relacionadas con la persistencia y valentía, en un extremo, y la amabilidad y el amor, en el otro.

Estas cuatro fortalezas tenían los valores más altos de discriminación. Es decir, los participantes en su mayoría reconocen un continuo de fortalezas en el continuo sí-mismo / otros. En un extremo se ubican las fortalezas vinculadas con la virtud coraje y en el otro extremo las vinculadas con la virtud de humanidad. Esto significa el reconocimiento de dos prototipos de individuos admirados. Uno de ellos estaría relacionado con la persistencia en el logro de las metas y la capacidad para hacer frente a la adversidad. El otro estaría en relación con la fidelidad a los principios, la lealtad, la solidaridad, el altruismo y la capacidad de amar. En relación con la clasificación de 24 fortalezas en 6 virtudes, los resultados de este estudio muestran que la mayoría de las fortalezas reconocidas por los participantes se ubican en relación con las virtudes de coraje y humanidad. La virtud justicia no aparece representada en estas agrupaciones.

La segunda dimensión resultó menos clara y explicaba el $25 \%$ de la inercia. Las fortalezas del carácter con mayor poder discriminante fueron perspectiva, humildad y creatividad, en un extremo, y la fortaleza de humor, en el otro. Esta agrupación parece representar el continuo reflexivo-expansivo. En un extremo se encontrarían ubicadas las fortalezas relacionadas con el razonamiento (p. ej., perspectiva y creatividad) y en el otro extremo estarían las vinculadas con los aspectos sociales, la alegría y el disfrute (e. g, amor, vitalidad). 
Tabla 2. Frecuencia de las fortalezas del carácter reconocidas en personajes admirados e historias positivas

\begin{tabular}{|c|c|c|c|c|c|}
\hline \multirow[t]{2}{*}{ Fortalezas } & \multirow[t]{2}{*}{ Virtudes } & \multicolumn{2}{|c|}{ Palabras } & \multicolumn{2}{|c|}{ Historias } \\
\hline & & $\mathrm{N}$ & Total \% & $\mathrm{N}$ & Total \% \\
\hline Integridad & Coraje & 142 & 37.6 & 24 & 6.49 \\
\hline Perseverancia & Coraje & 126 & 33.3 & 45 & 12.16 \\
\hline Bondad & Humanidad & 125 & 33.1 & 86 & 23.24 \\
\hline Amor & Humanidad & 72 & 19 & 71 & 19.19 \\
\hline Valentía & Coraje & 54 & 14.3 & 25 & 6.76 \\
\hline Humildad & Templanza & 53 & 14 & 6 & 1.62 \\
\hline Creatividad & Sabiduría & 36 & 9.5 & 11 & 2.97 \\
\hline Perspectiva & Sabiduría & 31 & 8.2 & 19 & 5.14 \\
\hline Humor & Trascendencia & 29 & 7.7 & 6 & 1.62 \\
\hline Vitalidad & Coraje & 26 & 6.9 & 5 & 1.35 \\
\hline Autorregulación & Templanza & 25 & 6.6 & 4 & 1.08 \\
\hline Liderazgo & Justicia & 15 & 4 & 11 & 2.97 \\
\hline Esperanza & Trascendencia & 13 & 3.4 & 7 & 1.89 \\
\hline Prudencia & Templanza & 10 & 2.6 & 4 & 1.08 \\
\hline Apertura & Sabiduría & 9 & 2.4 & - & - \\
\hline Amor por el Saber & Sabiduría & 6 & 1.6 & 5 & 1.35 \\
\hline Espiritualidad & Trascendencia & 4 & 1.1 & - & - \\
\hline Ciudadanía & Justicia & 3 & .8 & 4 & 1.08 \\
\hline Imparcialidad & Justicia & 3 & .8 & - & - \\
\hline Inteligencia Social & Sabiduría & 3 & .8 & 5 & 1.35 \\
\hline Clemencia & Templanza & 3 & .8 & 4 & 1.08 \\
\hline Curiosidad & Sabiduría & 2 & .5 & 3 & .81 \\
\hline Apreciación & Trascendencia & 1 & .3 & 4 & 1.08 \\
\hline
\end{tabular}

Nota. Apreciación = Apreciación de la belleza y de la excelencia; Ciudadanía = Ciudadanía o trabajo en equipo; Clemencia $=$ Clemencia y misericordia; Humildad $=$ Humildad/Modestia .

Estas dos dimensiones crean cuatro cuadrantes de fortalezas: a) Sí-Mismo/Expansivo (valentía, vitalidad y persistencia; b) Otro/Expansivo (amor, bondad e integridad); c) Sí-Mismo/Reflexivo (creatividad) y d) Otro/Reflexivo (humildad, perspectiva y autorregulación).

\section{Tipos de Personajes Admirados}

En cuanto a los personajes admirados, las fortalezas de carácter mencionadas se asociaron con los familiares $(32,3 \%)$, la población general $(20,6 \%)$ y los líderes espirituales (12,2\%) que en conjunto representan el $67 \%$ de los personajes prototípicos admirados seleccionados por los participantes. El resto de los personajes admirados tenía una frecuencia baja $(<8 \%)$. Con el objetivo de determinar cuáles fortalezas del carácter se asociaban a los prototipos admirados se realizó un análisis de correspondencias y se obtuvieron dos dimensiones, la primera de ellas explicaba un alto porcentaje de la inercia (45\%). Este análisis muestra que los personajes del entorno familiar y de la población general son admirados en relación con las fortalezas amor, integridad y persistencia; los líderes espirituales están en relación con las fortalezas de perspectiva, humildad, autorregulación y bondad. 
Figura 1. Análisis de correspondencias múltiples de las fortalezas del carácter más frecuentes

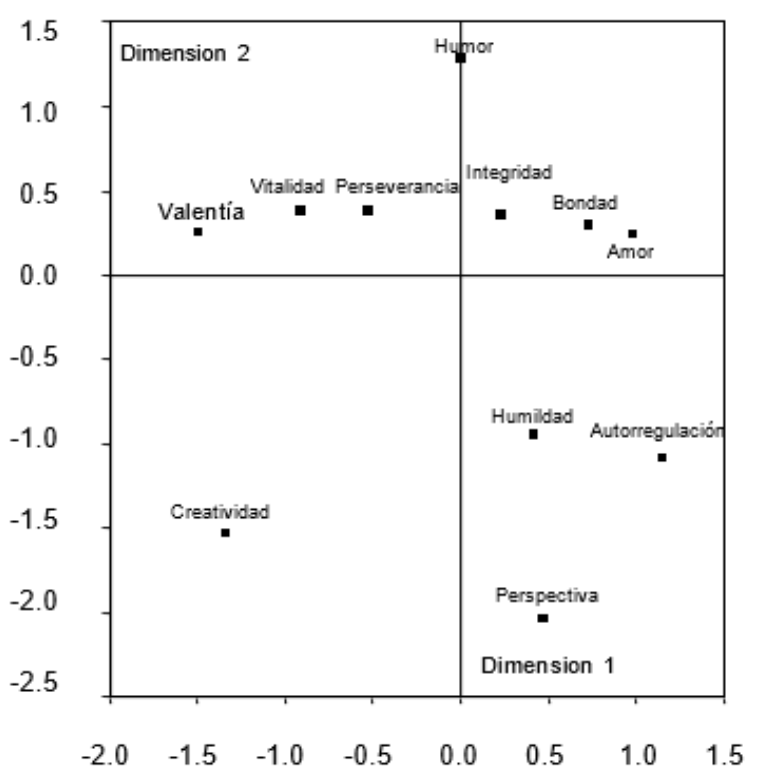

\section{Diferencias Individuales según Edad y Género}

En términos generales, no se encontraron diferencias individuales en las fortalezas reconocidas en los prototipos según edad y sexo. En cuanto al sexo, se encontraron diferencias en solo 4 de las 24 fortalezas de carácter analizadas. Las mujeres reconocen mayormente como características positivas la bondad $(19,8 \%$ vs. $13.2 \%, X 2=5.45, p<.05)$ y la esperanza $(2,9 \%$ vs. $\left.0.5 \%, x^{2}=4.75, p<.05\right)$ en comparación con los hombres. En cambio, los hombres reconocen como rasgos positivos en los demás las fortalezas de perspectiva $\left(5,6 \%\right.$ frente a $\left.2.6 \%, x^{2}=3,99, \mathrm{p}<.05\right)$ y el liderazgo $\left(3,2 \%\right.$ vs $\left.0.8 \%, X^{2}=4,80, p<.05\right)$ en comparación con sus pares femeninas. Con el fin de analizar la relación entre fortalezas del carácter y de la edad, se dividió a los participantes en dos grupos según la mediana de edad (mayores y menores de 41 años). Se encontraron diferencias en la fortaleza amor, que estaba presente en el grupo más joven comparado con el más grande. $(11,6 \%$ frente a $\left.7.4 \%, x^{2}=6,75, p<.05\right)$.

\section{Análisis de las Historias Positivas Análisis de Contenido}

Se codificó cada una de las historias en función de las fortalezas que aparecían mencionadas o a las que hacía referencia el contenido de la historia. Se usó un procedimiento similar al utilizado para codificar las palabras de los personajes admirados (Ver Tabla 1). Se utilizó una clasificación de categorías dicotómicas excluyentes para identificar el contenido dominante en cada historia. Un bajo porcentaje de las historias resultó inclasificable $(\mathrm{n}=$ $16,4.3 \%)$. Los resultados mostraron que las fortalezas del carácter con las frecuencias más altas eran la bondad y el amor, correspondientes a la virtud humanidad, seguido de las correspondientes a la virtud coraje, tales como la persistencia, la valentía y la honestidad. Estas fortalezas representaron el $68 \%$ de las clasificaciones realizadas en las historias. Las fortalezas del carácter menos frecuentes y, por lo tanto, menos representadas en el contenido de la historia fueron el liderazgo, la clemencia, la prudencia, la autorregulación, la apreciación de la belleza y la curiosidad. Este hallazgo resultó muy similar al obtenido con las características positivas de personajes admirados.

\section{Historias Positivas}

Dado que las narrativas proporcionan un contexto situacional en donde las historias se desarrollan, se llevó a cabo un análisis de contenido con el objetivo de relevar el tema más frecuente en cada relato. Se utilizó una metodología inductiva y sobre esa base se generaron diez categorías de códigos que eran mutuamente excluyentes. Cada historia fue reclasificada en categorías más estrechas, que representaban más claramente el núcleo temático de la historia. Las cuatro categorías finales fueron: a) Hacer frente a la adversidad en diversas situaciones ( $n=77,20.4 \%$ ); b) La fidelidad a los principios éticos ( $n=71,18.8 \%)$; c) Las relaciones de ayuda ( $n=108,27.8 \%)$ y d) Los intercambios de afecto ( $n=46,12.2 \%)$. Un bajo porcentaje de las historias resultó inclasificable $(n=32,8.5 \%)$. Otros temas que aparecieron en las historias estaban relacionados con el perdón, la obtención de placer en actividades solitarias y en grupo. Estos temas resultaron de muy baja frecuencia $(<2 \%)$.

\section{Tipo de Relación Predominante}

Al igual que en el análisis anterior, se realizó un análisis de contenido con el objetivo de caracterizar la naturaleza de la relación predominante en las historias. Siguiendo los mismos pasos que para el análisis anterior, se crearon cinco categorías de códigos a partir de las narrativas de los participantes. Las categorías de los códigos fueron: la familia, la amistad, la pareja, el trabajo/estudio y las relaciones con los extraños. Las historias fueron reclasificadas en función de los códigos generados. Los resultados muestran historias relacionadas con la familia $(n=$ $116,30.7 \%)$, los vínculos de amistad $(n=38,10 \%)$ y los vínculos con desconocidos ( $n=142,37.6 \%$ ). Estos tres tipos de relaciones representaron el $78,3 \%$ de las historias. Las historias relacionadas con la pareja y el estudio y/o trabajo tenían baja frecuencia $(n=17,4.5 \%$, y $n=34,9 \%$; respectivamente).

\section{Discusión}

El objetivo central de esta investigación fue analizar si la clasificación de fortalezas del carácter de Peterson y Seligman (2004) permitía captar las concepciones implícitas acerca de las virtudes y fortalezas en población general no consultante. En suma, la pregunta que guío esta investigación fue si la clasificación VIA, tal cual como fue diseñada por los autores, era apta para estudiar los rasgos positivos en una población latinoamericana.

Este estudio se inspiró en el trabajo de Park y Peterson (2006) con ocasión de solicitar a padres de niños descripciones de las virtudes más valoradas 
en los infantes y su posterior codificación por expertos mediante la clasificación VIA. Sin embargo, esta investigación se diferenció en varios aspectos. La principal estrategia utilizada para la recolección de datos fue un procedimiento de free listing (listas libres), metodología similar a la utilizada para el estudio acerca de la excelencia moral de Walker y Pitts (1998). Los autores consideraron que resultaba importante relevar las ideas acerca de la excelencia moral presentes en la vida cotidiana y que se encuentran insuficientemente representadas en las teorías psicológicas sobre el carácter y la excelencia moral. Coincidimos con Hart (1998) en que el punto de vista del sentido común, sin la opinión de algún tipo de juicio experto, no sería del todo adecuado. En consonancia, el presente estudio incluyó la codificación de las respuestas libres de los participantes en función de la clasificación de veinticuatro fortalezas de carácter propuestas por Peterson y Seligman (2004), para complementar el punto de vista de las personas comunes.

Los resultados de este estudio tienen una implicación cultural respecto de la clasificación VIA. En primer lugar, algunas fortalezas aparecen subrepresentadas y algunas de ellas no aparecen ni siquiera mencionadas por los participantes cuando se les pide que describan los rasgos positivos de sus personajes admirados (p. ej. espiritualidad, perdón, apreciación de la belleza). Las altas frecuencias de ciertas virtudes del carácter representan un consenso cultural compartido en relación con los rasgos positivos más relevantes para la cultura en particular de la que se trate, en este caso, la Argentina (Quinlan, 2005). Aquellas fortalezas con muy baja frecuencia estarían representando un punto de vista idiosincrático de algunos individuos de esa cultura.

Estos hallazgos están en relación con los resultados derivados de la aplicación empírica del cuestionario de virtudes y fortalezas basado en la clasificación VIA en diferentes países alrededor del globo. En términos generales la agrupación propuesta de 24 fortalezas en 6 virtudes no se cumplió en casi ningún caso. Esto estaría indicando importantes diferencias culturales en cómo las personas describen sus virtudes y la relación entre ellas, cuestionando seriamente el carácter universal de la clasificación.

Este hallazgo está en consonancia con otros estudios transculturales que abordaron este problema (Bardar \& Kashdan, 2010; Duan et al., 2012; Khumalo, Wissing \& Temane, 2008; LittmanOvadia \& Lavy, 2012; Macdonald, Bore \& Munro, 2008; Singh \& Choubisa, 2010). Asimismo, en países tan distintos como China, EEUU o Argentina, los resultados de las técnicas de análisis factorial sobre la mencionada clasificación revelaron que esa clasificación no tiene validez empírica desde el punto de vista de su estructura interna (Cosentino, 2011; Duan et al., 2012; Peterson \& Seligman, 2004). Esta situación parece sugerir que en las diferentes culturas existen relaciones estructurales diversas respecto de los constructos éticos impuestos.
Asimismo, el análisis de las palabras que caracterizaban los rasgos positivos de las personas admiradas dio como resultado dos dimensiones a) Sí-mismo vs. Otros y b) Reflexivo vs. Expansivo. En términos generales, esta agrupación es similar a los resultados obtenidos por Peterson (2006) con ocasión de analizar la agrupación de las fortalezas del carácter. El autor había propuesto un modelo circumplejo bidimensional que constaba de dos dimensiones, la primera de naturaleza más social: Foco en Sí-Mismo vs Foco en los Otros y la segunda que tenía los polos Mente-Corazón; es decir, que consideraba las fortalezas más relacionadas con la restricción intelectual en un polo (p. ej. perspectiva, autorregulación) y las de la expresión emocional en el otro (p. ej. gratitud, amor).

La agrupación propuesta de dos dimensiones y cuatro cuadrantes no pretende proporcionar una organización definida de las fortalezas del carácter, sino que tiene por objetivo contribuir a una perspectiva multicultural sobre la organización de los rasgos positivos surgidos de la clasificación VIA. Como se ha comentado, los diferentes estudios transculturales sobre la clasificación VIA han propuesto agrupaciones alternativas a la original de Peterson y Seligman, en función de los resultados derivados de las investigaciones. Así, por ejemplo, Shryack, Steger, Krueger y Kallie (2010) proponen que las fortalezas se agrupen en virtudes intelectuales, interpersonales y templanza (i. e., de la autorregulación); Duan et al. (2012) proponen las agrupaciones de vitalidad, interpersonales y de cautela utilizando una muestra de población China y Cosentino (2011) propuso progresar, fraternizar y moderarse. Estos modelos resultan más sensibles culturalmente y están en la línea de los resultados obtenidos en esta investigación.

En este contexto, deben tomarse en cuenta las diferencias entre las metodologías empleadas. En primer lugar, el desarrollo de la clasificación VIA de las fortalezas del carácter tuvo una estrategia topdown. Se estudiaron las tradiciones filosóficas y religiosas más influyentes, se derivaron 6 virtudes nucleares y, como paso posterior, los teóricos y académicos generaron teóricamente 24 fortalezas que se relacionarían con esas 6 virtudes. Sin embargo, los estudios empíricos que mostraron un pobre ajuste de los datos al modelo teórico original $24 / 6$ de la clasificación VIA utilizaron una metodología muy distinta de características bottomup, dando énfasis a las agrupaciones que las personas reconocían en diferentes contextos culturales (p. ej., Cosentino; Shryack et al., 2010). En segundo lugar, Peterson y Seligman habían señalado el carácter provisional de la clasificación VIA y afirmaban que esta clasificación podría ser cambiada, reducida o ampliada en función de los avances empíricos de la disciplina (Peterson, 2006).

En consecuencia, la presente investigación en la misma línea de lo señalado por otros estudios culturales sobre las fortalezas del carácter, indica la necesidad de ajustar la clasificación VIA en función de las variaciones culturales ya apuntadas por estas investigaciones. Asimismo, esta investigación tiene 
importantes implicaciones para las tareas en las que tengan que aplicarse intervenciones con clientes de diferentes backgrounds culturales. Como se mostró en esta investigación, las personas no reconocen o reconocen limitadamente la totalidad de fortalezas señaladas por la clasificación original de Peterson y Seligman. Las clasificaciones que proponen modelos alternativos y/o que proponen resultados divergentes con el modelo original (precisamente por la inclusión de las variables culturales) parece que no han podido contribuir a generar los cambios necesarios sobre la clasificación en su formato original.

Por lo tanto, al menos desde el punto de vista cultural, y, más aún, de cara a las intervenciones clínicas, la clasificación de Peterson y Seligman (2004) debería ser revisada o bien ajustada en función de las variables culturales. Por ejemplo, un psicólogo puede estar trabajando con sus clientes sobre el desarrollo de alguna fortaleza del carácter en particular (p. ej., gratitud), pero en el contexto cultural del cliente esa fortaleza del carácter tiende a ser socialmente irrelevante. En consecuencia, dicha intervención puede tropezar con obstáculos surgidos desde marco conceptual utilizado, en razón de su inconsistencia con el background cultural del cliente. En las intervenciones psicológicas ajustadas culturalmente, las fortalezas del carácter a utilizar deberían ser principalmente provenientes del conjunto de fortalezas que las personas de un determinado grupo cultural reconocen socialmente. Orientar la intervención a las fortalezas del carácter culturalmente relevantes podría resultar beneficioso para el objetivo de la intervención propuesto.

Una mayor sensibilidad cultural aplicada a la utilización de las fortalezas del carácter como herramienta de cambio psicológico, puede dar lugar a intervenciones específicas más ajustadas culturalmente y con implicaciones más positivas para los clientes, en comparación con intervenciones ético-impuestas. Se sugiere la necesidad de hacer más investigaciones sobre los aspectos émicos de las fortalezas del carácter. Las intervenciones culturalmente sensibles podrían beneficiarse de ese importante avance, generando consecuencias positivas que afirmen de forma implícita o explícita la identidad cultural de los clientes de grupos culturales diversos.

\section{Referencias}

Bardar, I. \& Kashdan, T. (2010). Character strengths and well-being in Croatia: An empirical investigation of structure and correlates. Journal of Research in Personality, 44(1), 151-154. doi:10.1016/j.jrp.2009.12.001

Berry, H. (1969). On cross-cultural comparability. International Journal of Psychology, 4(2), 119128. doi:10.1080/00207596908247261

Berry, J. (1989). Imposed etics, emics, derived etics. International Journal of Psychology, 24(6), 721735. doi:10.1080/00207598908247841

Berry, J., Poortinga, Y., Segall, M. \& Dasen, P. (2006). Cross-cultural psychology: Research and applications. New York: Cambridge University Press.

Castro Solano, A. (Ed.). (2014). Positive Psychology in Latin America. Springer, Dordrecht: Springer Netherlands. doi:10.1007/978-94-017-9035-2

Casullo, M. \& Fernández, M. (2006). Las propuestas de La psicología positiva: ¿Universales psicológicos o particulares de una visión cultural? Anuario de Investigaciones, 14(25), 261-268. Recuperado de http://www.scielo.org.ar/pdf/anuinv/v14/v14a25. $\underline{\mathrm{pd}}$

Cosentino, A. (2009). Evaluación de las virtudes y fortalezas humanas en población de habla hispana. Psicodebate, 10, 53-71. doi:10.18682/pd.v10i0.388

Cosentino, A. (2011). Fortalezas del carácter en militares argentinos (Tesis doctoral inédita, Universidad de Palermo, Buenos Aires, Argentina).

Cosentino, A. (2014). Character strengths: Measurement and studies in Argentina with military and general population samples. En A. Castro Solano (Ed.), Positive Psychology in Latin America (pp. 111127). Dordrecht: Springer Netherlands. doi:10.1007/978-94-017-9035-2_6.

Cosentino, A. \& Castro Solano, A. (2012). Character Strengths: A Study of Argentinean Soldiers. The Spanish Journal of Psychology, 15(1), 199-215. doi:10.5209/rev SJOP.2012.v15.n1.37310

Cosentino, A. \& Castro Solano, A. (2014). The assessment of multicultural strength: Design and validation of an openness to the other affective domain inventory. European Journal of Psychological Assessment, 30(4), 261-273. doi:10.1027/10155759/a000187

Christopher, J. \& Hickinbottom, S. (2008). Positive psychology, ethnocentrism, and the disguised ideology of individualism. Theory \& Psychology, 18(5), 563-589. doi:10.1177/0959354308093396

Dahlsgaard, K., Peterson, C. \& Seligman, M. (2005). Shared virtue: The convergence of valued human strengths across culture and history. Review of General Psychology, 9(3), 203-213. doi:10.1037/1089-2680.9.3.203

Davidson, A., Jaccard, J., Triandis, H., Morales, M. \& DíazGuerrero, R. (1976). Cross-cultural model testing: Toward a solution of the etic-emic dilemma. International Journal of Psychology, 11(1), 1-13. doi:10.1080/00207597608247343

Duan, W., Ho, S., Yu, B., Tang, X., Zhang, Y., Li, T. \& Yuen, T. (2012). Factor structure of the Chinese virtues questionnaire. Research on Social Work $\begin{array}{ll}\text { Practice, 22(6), } & \text { 680-688. }\end{array}$ doi:10.1177/1049731512450074

Fowler, R., Seligman, M. \& Koocher, G. (1999). The APA 1998 annual report. American Psychologist, 54(8), 537-568. doi:10.1037/0003066X.54.8.537

Hart, D. (1998). Can prototypes inform moral developmental theory? Developmental 
Psychology, 34(3), 420-423. doi:10.1037/00121649.34.3.420

Helfrich, H. (1999). Beyond the dilemma of cross-cultural psychology: Resolving the tension between etic and emic approaches. Culture \& Psychology, 5(2), 131-153. doi:10.1177/1354067X9952002

Kagitcibasi, C. \& Berry, J. (1989). Cross-cultural psychology: Current research and trends. Annual review of psychology, 40(1), 493-531. doi:10.1146/annurev.ps.40.020189.002425

Khumalo, I., Wissing, M. \& Temane, Q. (2008). Exploring the validity of the Values-In-Action Inventory of Strengths (VIA-IS) in an African context. Journal of Psychology in Africa, 18(1), 133-142. Recuperado de http://dspace.nwu.ac.za/bitstream/handle/10394 1721/khumalo itumelengp.pdf; sequence $=1$

Lambert, L., Pasha-Zaidi, N., Passmore, H. \& Al-Karam, C. (2015). Developing an indigenous positive psychology in the United Arab Emirates. Middle East Journal of Positive Psychology, 1(1), 1-23. Recuperado http://www. middleeastjournalofpositivepsycholo gy.org/index.php/meipp/article/view/24

Linley, P., Joseph, S., Harrington, S. \& Wood, A. (2006). Positive psychology: Past, present, and (possible) future. The Journal of Positive Psychology, 1(1), 3-16. doi:10.1080/17439760500372796

Littman-Ovadia, H. \& Lavy, S. (2012). Character strengths in Israel: Hebrew adaptation of the VIA Inventory of Strengths. European Journal of Psychological Assessment, 28(1), 41-50. doi:10.1027/10155759/a000089

López, S., Prosser, E., Edwards, L., Magyar-Moe, J., Neufeld, J. \& Rasmussen, H. (2002). Putting positive psychology in a multicultural context. En C. Snyder \& S. López (Eds.), Handbook of positive psychology (pp. 700-714). New York: Oxford University Press.

Lounsbury, J., Fisher, L., Levy, J. \& Welsh, D. (2009). An investigation of character strengths in relation to the academic success of college students. Individual Differences Research, 7(1), 52-69. Recuperado http://citeseerx.ist.psu.edu/viewdoc/download?d oi=10.1.1.453.9217\&rep=rep1\&type=pdf

Macdonald, C., Bore, M. \& Munro, D. (2008). Values in action scale and the Big 5: An empirical indication of structure. Journal of Research in Personality, 42(4), 787-799. doi:10.1016/j.jp.2007.10.003

Park, N. \& Peterson, C. (2009). Strengths of character in schools. En R. Gilman, E. Huebner \& M. Furlong (Eds.), Handbook of positive psychology in schools (pp. 65-76). Nueva York: Routledge.

Park, N., Peterson, C. \& Seligman, M. (2004). Strengths of character and well-being. Journal of Social and Clinical Psychology, 23(5), 603-619. Recuperado de $\quad$ http://www.viacharacter.org/blog/wpcontent/uploads/2013/12/Character-strengthswell-being-Park-Peterson-Seligman-2004.pdf

Peterson, C. (2006). A primer in positive psychology. New York: Oxford University Press.
Peterson, C. \& Park, N. (2009). Classifying and measuring strengths of character. En C. Snyder \& S. Lopez (Eds.), The Oxford handbook of positive psychology (2a ed.). Oxford: Oxford University Press. 10.1093/oxfordhb/9780195187243.013.0004

Peterson, C. \& Seligman, M. (2004). Character strengths and virtues: $A$ handbook and classification. Washington: APA Press and Oxford University Press.

Peterson, C., Park, N. \& Seligman M. (2006). Greater strengths of character and recovery from illness. The Journal of Positive Psychology, 1(1), 17-26. doi:10.1080/17439760500372739

Peterson, C., Park, N., Pole, N., D'Andrea, W. \& Seligman, M. (2008). Strengths of character and posttraumatic growth. Journal of Traumatic Stress, 21(2), 214-217. doi:10.1002/jts.20332

Quinlan, M. (2005). Considerations for collecting Freelists in the field: Examples from ethobotany. Field Methods, 17(3), 219-234. doi:10.1177/1525822X05277460

Seligman, P. \& Csíkszentmihályi, M. (2000). Positive psychology: An introduction. American Psychologist, 55(1), 5-14. doi:10.1037//0003066X.55.1.5

Shryack, J., Steger, M., Krueger, R. \& Kallie, C. (2010). The Structure of virtue: An empirical investigation of the dimensionality of the virtues in action inventory of strengths. Personality and Individual Differences, 48(6), 714-719. doi:10.1016/j.paid.2010.01.007

Singh, K. \& Choubisa, R. (2010). Empirical validation of values in action-inventory of strengths (VIA-IS) in Indian context. Psychological Studies, 55(2), 151-158. doi:10.1007/s12646-010-0015-4

Sinha, D. (1997). Indiginizing psychology. En J. Berry, Y. Poortinga \& J. Pandley (Eds.), Handbook of cross-cultural psychology: Theory and method (Vol. 1, 2a ed., pp. 129-169). Boston: Allyn and Bacon.

Snyder, C., López, S. \& Pedrotti, J. (2011). Positive psychology: The scientific and practical explorations of human strengths ( $2 \mathrm{a} \quad \mathrm{ed}$.$) .$ Thousand Oaks: Sage Publications, Inc.

Steger, M., Hicks, B., Kashdan, T., Krueger, R. \& Bouchard, T. (2007). Genetic and environmental influences on the positive traits of the Values in Action classification, and biometric covariance with normal personality. Journal of Research in Personality, 41(3), 524-539. Recuperado de http://mason.gmu.edu/ tkashdan/publications/g enetics positivetraits.pdf

Triandis, H., Malpas, R. \& Davidson, A. (1973). Psychology and culture. Annual Review of Psychology, 24(1), 355-

378.doi:10.1146/annurev.ps.24.020173.002035

Walker, L. \& Pitts, R. (1998). Naturalistic conceptions of moral maturity. Developmental psychology, 34(3), 403. doi:10.1037/0012-1649.34 
VIRTUDES Y FORTALEZAS DEL CARÁCTER EN POBLACIÓN ADULTA DE BUENOS AIRES 